\title{
SET DIFFERENTIAL EQUATIONS WITH CAUSAL OPERATORS
}

\author{
Z. DRICI, F. A. MCRAE, AND J. VASUNDHARA DEVI
}

Received 21 October 2004

We obtain some basic results on existence, uniqueness, and continuous dependence of solutions with respect to initial values for set differential equations with causal operators.

\section{Introduction}

Differential equations involving causal operators have gained much attention of late and some results are assembled in a recent monograph [1]. The term causal is adopted from the engineering literature. Basically, a causal operator is a nonanticipative operator. The theory of these operators has the powerful quality of unifying ordinary differential equations, integrodifferential equations, differential equations with finite or infinite delay, Volterra integral equations, and neutral functional equations, to name a few.

The study of set differential equations (SDE) in a metric space is interesting due to its applicability to multivalued differential inclusions and fuzzy differential equations and its inclusion of ordinary differential systems as a special case $[2,4]$.

A combination of these two concepts leads to set differential equations with causal operators. In this paper, using this setup, we obtain some basic results on existence, uniqueness, and continuous dependence of solutions with respect to initial values.

\section{Preliminaries}

Let $K_{c}\left(\mathbb{R}^{n}\right)$ denote the collection of all nonempty compact and convex subsets of $\mathbb{R}^{n}$. Define the Hausdorff metric

$$
D[A, B]=\max \left[\sup _{x \in B} d(x, A), \sup _{y \in A} d(y, B)\right],
$$

where $A, B$ are bounded sets in $\mathbb{R}^{n}$ and $d(x, A)=\inf [d(x, y): y \in A]$. We observe that $K_{c}\left(\mathbb{R}^{n}\right)$ is a complete metric space.

Suppose that the space $K_{c}\left(\mathbb{R}^{n}\right)$ is equipped with the natural algebraic operations of addition and nonnegative scalar multiplication. Then, $K_{c}\left(\mathbb{R}^{n}\right)$ becomes a semilinear metric space, which can be embedded as a complete cone into a corresponding Banach space. 
We note that the Hausdorff metric (2.1) satisfies the following properties:

$$
\begin{gathered}
D[A+C, B+C]=D[A, B], \quad D[A, B]=D[B, A], \\
D[\lambda A, \lambda B]=\lambda D[A, B], \\
D[A, B] \leq D[A, C]+D[C, B]
\end{gathered}
$$

for all $A, B, C \in K_{c}\left(\mathbb{R}^{n}\right)$ and $\lambda \in \mathbb{R}_{+}$.

Given any two sets $A, B \in K_{c}\left(\mathbb{R}^{n}\right)$ if there exists a set $C \in K_{c}\left(\mathbb{R}^{n}\right)$ satisfying $A=B+C$, then $A-B$ is defined as the Hukuhara difference of the sets $A$ and $B$.

The mapping $F: I \rightarrow K_{c}\left(\mathbb{R}^{n}\right)$ has a Hukuhara derivative $D_{H} F\left(t_{0}\right)$ at a point $t_{0} \in I$, if

$$
\lim _{h \rightarrow 0^{+}} \frac{F\left(t_{0}+h\right)-F\left(t_{0}\right)}{h}, \quad \lim _{h \rightarrow 0^{+}} \frac{F\left(t_{0}\right)-F\left(t_{0}-h\right)}{h}
$$

exist in the topology of $K_{c}\left(\mathbb{R}^{n}\right)$ and are equal to $D_{H} F\left(t_{0}\right)$. Here $I$ is any interval in $\mathbb{R}$.

Now we can consider the set differential equation

$$
D_{H} U=F(t, U), \quad U\left(t_{0}\right)=U_{0} \in K_{c}\left(\mathbb{R}^{n}\right), \quad t_{0} \geq 0,
$$

where $F \in C\left[\mathbb{R}_{+} \times K_{c}\left(\mathbb{R}^{n}\right), K_{c}\left(\mathbb{R}^{n}\right)\right]$.

Definition 2.1. The mapping $U \in C^{1}\left[J, K_{c}\left(\mathbb{R}^{n}\right)\right], J=\left[t_{0}, t_{0}+a\right]$, is said to be a solution of (2.6) on $J$ if it satisfies (2.6) on $J$.

Since $U(t)$ is continuously differentiable, we have

$$
U(t)=U_{0}+\int_{t_{0}}^{t} D_{H} U(s) d s, \quad t \in J .
$$

Hence, we can associate with the IVP (2.6) the Hukuhara integral

$$
U(t)=U_{0}+\int_{t_{0}}^{t} F(s, U(s)) d s, \quad t \in J .
$$

The following properties are useful tools in proving theorems in the SDE setup. If $F:\left[t_{0}, T\right] \rightarrow K_{c}\left(\mathbb{R}^{n}\right)$ is integrable, we have

$$
\begin{gathered}
\int_{t_{0}}^{t_{2}} F(t) d t=\int_{t_{0}}^{t_{1}} F(t) d t+\int_{t_{1}}^{t_{2}} F(t) d t, \quad t_{0} \leq t_{1} \leq t_{2} \leq T, \\
\int_{t_{0}}^{T} \lambda F(t) d t=\lambda \int_{t_{0}}^{T} F(t) d t, \quad \lambda \in \mathbb{R}_{+} .
\end{gathered}
$$

Also, if $F, G:\left[t_{0}, T\right] \rightarrow K_{c}\left(\mathbb{R}^{n}\right)$ are integrable, then $D[F(\cdot), G(\cdot)]:\left[t_{0}, T\right] \rightarrow \mathbb{R}$ is integrable and

$$
D\left[\int_{t_{0}}^{t} F(s) d s, \int_{t_{0}}^{t} G(s) d s\right] \leq \int_{t_{0}}^{t} D[F(s), G(s)] d s .
$$


We observe that

$$
D[A, \theta]=\|A\|=\sup _{a \in A}\|a\|
$$

for $A \in K_{c}\left(\mathbb{R}^{n}\right)$, where $\theta$ is the zero element of $\mathbb{R}^{n}$, which is regarded as a one-point set.

\section{Main results}

We will devote this section to extend certain basic results to SDEs with causal or nonanticipative maps of Volterra type, since such equations provide a unified treatment of the basic theory of SDEs, SDEs with delay and set integrodifferential equations which in turn include ordinary dynamic systems of the corresponding types.

Let $E=C\left[\left[t_{0}, T\right], K_{c}\left(\mathbb{R}^{n}\right)\right]$ with norm

$$
D_{0}[U, \theta]=\sup _{t_{0} \leq t \leq T} D[U(t), \theta] .
$$

Definition 3.1. Suppose that $Q \in C[E, E]$, then $Q$ is said to be a causal map or a nonanticipative map if $U(s)=V(s), t_{0} \leq s \leq t \leq T$, where $U, V \in E$, then $(Q U)(s)=(Q V)(s)$, $t_{0} \leq s \leq t$.

We define the IVP for an SDE with causal map, using the Hukuhara derivative as follows:

$$
D_{H} U(t)=(Q U)(t), \quad U\left(t_{0}\right)=U_{0} \in K_{c}\left(\mathbb{R}^{n}\right) .
$$

Before we proceed to prove an existence and uniqueness result for (3.2), we need the following comparison results.

Theorem 3.2. Assume that $m \in C\left[J, \mathbb{R}_{+}\right], g \in C\left[J \times \mathbb{R}_{+}, \mathbb{R}_{+}\right]$and, for $t \in J=\left[t_{0}, T\right]$,

$$
D_{-} m(t) \leq g\left(t,|m|_{0}(t)\right) \text {, }
$$

where $|m|_{0}(t)=\sup _{t_{0} \leq s \leq t}|m(s)|$. Suppose that $r(t)=r\left(t, t_{0}, w_{0}\right)$ is the maximal solution of the scalar differential equation

$$
w^{\prime}=g(t, w), \quad w\left(t_{0}\right)=w_{0} \geq 0,
$$

existing on $J$. Then, $m\left(t_{0}\right) \leq w_{0}$ implies $m(t) \leq r(t), t \in J$.

Proof. To prove the stated inequality, it is enough to prove that

$$
m(t)<w\left(t, t_{0}, w_{0}, \epsilon\right), \quad t \geq t_{0}, t \in J,
$$

where $w\left(t, t_{0}, w_{0}, \epsilon\right)$ is any solution of

$$
w^{\prime}=g(t, w)+\epsilon, \quad w\left(t_{0}\right)=w_{0}+\epsilon, \quad \epsilon>0,
$$

since $\lim _{\epsilon \rightarrow 0^{+}} w\left(t, t_{0}, w_{0}, \epsilon\right)=r\left(t, t_{0}, w_{0}\right)$. 
If (3.5) is not true, there exists a $t_{1}>t_{0}$ such that $m\left(t_{1}\right)=w\left(t_{1}, t_{0}, w_{0}, \epsilon\right)$ and $m(t)<$ $w\left(t, t_{0}, w_{0}, \epsilon\right), t_{0} \leq t<t_{1}$, in view of the fact $m\left(t_{0}\right)<w_{0}+\epsilon$.

Hence,

$$
D_{-} m\left(t_{1}\right) \geq w^{\prime}\left(t_{1}, t_{0}, w_{0}, \epsilon\right)=g\left(t_{1}, w\left(t_{1}, t_{0}, w_{0}, \epsilon\right)\right)+\epsilon .
$$

Now $g(t, w) \geq 0$ implies that $w\left(t, t_{0}, w_{0}, \epsilon\right)$ is nondecreasing in $t$, and this gives

$$
|m|_{0}\left(t_{1}\right)=w\left(t_{1}, t_{0}, w_{0}, \epsilon\right)=m\left(t_{1}\right)
$$

which in turn yields

$$
D_{-} m\left(t_{1}\right) \leq g\left(t_{1},|m|_{0}\left(t_{1}\right)\right)=g\left(t_{1}, w\left(t_{1}, t_{0}, w_{0}, \epsilon\right)\right)
$$

which is a contradiction to (3.7). Hence the theorem follows.

Next we obtain an estimate of the distance between any two solutions of (3.2) in terms of the maximal solution of (3.4) utilizing Theorem 3.2.

We define $D_{0}[U, V](t)=\max _{t_{0} \leq s \leq t} D[U(s), V(s)]$.

Theorem 3.3. Let $Q \in C[E, E]$ be a causal map such that for $t \in J$,

$$
D[(Q U)(t),(Q V)(t)] \leq g\left(t, D_{0}[U, V](t)\right),
$$

where $g \in C\left[J \times \mathbb{R}_{+}, \mathbb{R}_{+}\right]$. Suppose further that the maximal solution $r\left(t, t_{0}, w_{0}\right)$ of the differential equation (3.4) exists on $J$. Then, if $U(t), V(t)$ are any two solutions of (3.2) through $U\left(t_{0}\right)=U_{0}, V\left(t_{0}\right)=V_{0}, U_{0}, V_{0} \in K_{c}\left(\mathbb{R}^{n}\right)$ on $J$, respectively,

$$
D[U(t), V(t)] \leq r\left(t, t_{0}, w_{0}\right), \quad t \in J,
$$

provided that $D\left[U_{0}, V_{0}\right] \leq w_{0}$.

Proof. Set $m(t)=D[U(t), V(t)]$. Then $m\left(t_{0}\right)=D\left[U_{0}, V_{0}\right] \leq w_{0}$. Now for small $h>0, t \in$ $J$, consider $m(t+h)=D[U(t+h), V(t+h)]$. Using the property (2.4) of the Hausdorff metric $D$, we successively get the following relations:

$$
\begin{aligned}
m(t+h) \leq & D[U(t+h), U(t)+h(Q U)(t)] \\
& +D[U(t)+h(Q U)(t), V(t+h)] \leq D[U(t+h), U(t)+h(Q U)(t)] \\
& +D[U(t)+h(Q U)(t), V(t)+h(Q V)(t)]+D[V(t)+h(Q V)(t), V(t+h)] \\
\leq & D[U(t+h), U(t)+h(Q U)(t)]+D[U(t)+h(Q U)(t), U(t)+h(Q V)(t)] \\
& +D[U(t)+h(Q V)(t), V(t)+h(Q V)(t)]+D[V(t)+h(Q V)(t), V(t+h)] .
\end{aligned}
$$

Next, using the property (2.2) of the Hausdorff metric $D$ and the fact that the Hukuhara differences $U(t+h)-U(t)$ and $V(t+h)-V(t)$ exist for small $h>0$, we arrive at

$$
\begin{aligned}
m(t+h) \leq & D[U(t)+Z(t, h), U(t)+h(Q U)(t)]+D[h(Q U)(t), h(Q V)(t)] \\
& +D[U(t), V(t)]+D[V(t)+h(Q V)(t), V(t)+Y(t, h)],
\end{aligned}
$$


where $U(t+h)=U(t)+Z(t, h)$ and $V(t+h)=V(t)+Y(t, h)$. Again the property (2.2) gives

$$
\begin{aligned}
m(t+h) \leq & D[Z(t, h), h(Q U)(t)]+D[h(Q U)(t), h(Q V)(t)] \\
& +D[U(t), V(t)]+D[h(Q V)(t), Y(t, h)] .
\end{aligned}
$$

Since the Hukuhara differences exist, we can replace $Z(t, h)$ and $Y(t, h)$ with $U(t+h)-$ $U(t)$ and $V(t+h)-V(t)$, respectively. This gives, on subtracting $m(t)$ and dividing both sides with $h>0$,

$$
\begin{aligned}
\frac{m(t+h)-m(t)}{h} \leq & D\left[\frac{U(t+h)-U(t)}{h},(Q U)(t)\right]+D[(Q U)(t),(Q V)(t)] \\
& +D\left[(Q V)(t), \frac{V(t+h)-V(t)}{h}\right] .
\end{aligned}
$$

Now, taking limit supremum as $h \rightarrow 0^{+}$and using the fact that $U(t)$ and $V(t)$ are solutions of (3.2), along with the assumption (3.10) we obtain

$$
D^{+} m(t) \leq D[(Q U)(t),(Q V)(t)] \leq g\left(t, D_{0}[U, V](t)\right)=g\left(t,|m|_{0}(t)\right), \quad t \in J .
$$

Theorem 3.2 now guarantees the stated conclusion and the proof is complete.

Corollary 3.4. Let $Q \in C[E, E]$ be a causal map such that

$$
D[(Q U)(t), \theta] \leq g\left(t, D_{0}[U, \theta](t)\right),
$$

where $g \in C\left[J \times \mathbb{R}_{+}, \mathbb{R}_{+}\right]$. Also, suppose that $r\left(t, t_{0}, w_{0}\right)$ is the maximal solution of the scalar differential equation (3.4). Then, if $U\left(t, t_{0}, U_{0}\right)$ is any solution of (3.2) through $\left(t_{0}, U_{0}\right)$ with $U_{0} \in K_{c}\left(\mathbb{R}^{n}\right), D\left[U_{0}, \theta\right] \leq w_{0}$ implies $D[U(t), \theta] \leq r\left(t, t_{0}, w_{0}\right), t \in J$.

We begin by proving a local existence result using successive approximations.

Theorem 3.5. Assume that

(a) $Q \in C[B, E]$ is a causal map, where $B=B\left(U_{0}, b\right)=\left\{U \in E: D_{0}\left[U, U_{0}\right] \leq b\right\}$ and $D_{0}[(Q U), \theta](t) \leq M_{1}$, on $B$

(b) $g \in C\left[J \times[0,2 b], \mathbb{R}_{+}\right], g(t, w) \leq M_{2}$ on $J \times[0,2 b], g(t, 0) \equiv 0, g(t, w)$ is nondecreasing in $w$ for each $t \in J$ and $w(t)=0$ is the only solution of

$$
w^{\prime}=g(t, w), \quad w\left(t_{0}\right)=0 \quad \text { on } J
$$

(c) $D[(Q U)(t),(Q V)(t)] \leq g\left(t, D_{0}[U, V](t)\right)$ on $B$.

Then, the successive approximations defined by

$$
U_{n+1}(t)=U_{0}+\int_{t_{0}}^{t}\left(Q U_{n}\right)(s) d s, \quad n=0,1,2, \ldots,
$$

exist on $J_{0}=\left[t_{0}, t_{0}+\eta\right)$, where $\eta=\min \left[T-t_{0}, b / M\right]$ and $M=\max \left(M_{1}, M_{2}\right)$, and converge uniformly to the unique solution $U(t)$ of (3.2). 
Proof. For $t \in J_{0}$, we have, by induction, using property (2.2) and (2.10) of the Hausdorff metric $D$,

$$
\begin{aligned}
D\left[U_{n+1}(t), U_{0}\right] & =D\left[U_{0}+\int_{t_{0}}^{t}\left(Q U_{n}\right)(s) d s, U_{0}\right]=D\left[\int_{t_{0}}^{t}\left(Q U_{n}\right)(s) d s, \theta\right] \\
& \leq \int_{t_{0}}^{t} D\left[\left(Q U_{n}\right)(s), \theta\right] d s \\
& \leq \int_{t_{0}}^{t} D_{0}\left[Q U_{n}, \theta\right](t) d s \leq M_{1}\left(t-t_{0}\right) \leq M\left(t-t_{0}\right) \leq b,
\end{aligned}
$$

which shows the successive approximations are well defined on $J_{0}$.

Next, we define successive approximations for the problem (3.18) as follows:

$$
\begin{gathered}
w_{0}(t)=M\left(t-t_{0}\right), \\
w_{n+1}(t)=\int_{t_{0}}^{t} g\left(s, w_{n}(s)\right) d s, \quad t \in J_{0}, n=0,1,2, \ldots
\end{gathered}
$$

Then,

$$
w_{1}(t)=\int_{t_{0}}^{t} g\left(s, w_{0}(s)\right) d s \leq M_{2}\left(t-t_{0}\right) \leq M\left(t-t_{0}\right)=w_{0}(t) .
$$

Assume, for some $k>1, t \in J_{0}$, that

$$
w_{k}(t) \leq w_{k-1}(t)
$$

Then, using the monotonicity of $g$, we get

$$
w_{k+1}(t)=\int_{t_{0}}^{t} g\left(s, w_{k}(s)\right) d s \leq \int_{t_{0}}^{t} g\left(s, w_{k-1}(s)\right) d s=w_{k}(t) .
$$

Hence, the sequence $\left\{w_{k}(t)\right\}$ is monotone decreasing.

Since $w_{k}^{\prime}(t)=g\left(t, w_{k-1}(t)\right) \leq M_{2}, t \in J_{0}$, we conclude by Ascoli-Arzela theorem and the monotonicity of the sequence $\left\{w_{k}(t)\right\}$ that

$$
\lim _{t \rightarrow \infty} w_{n}(t)=w(t)
$$

uniformly on $J_{0}$. Since $w(t)$ satisfies (3.18), we get from condition (b) that $w(t) \equiv 0$ on $J_{0}$. Observing that for each $t \in J_{0}, t_{0} \leq s \leq t$,

$$
\begin{aligned}
D\left[U_{1}(s), U_{0}\right] & =D\left[U_{0}+\int_{t_{0}}^{s}\left(Q U_{0}\right)(\xi) d \xi, U_{0}\right]=D\left[\int_{t_{0}}^{s}\left(Q U_{0}\right)(\xi) d \xi, \theta\right] \\
& \leq \int_{t_{0}}^{s} D\left[\left(Q U_{0}\right)(\xi), \theta\right] d \xi \leq D_{0}\left[\left(Q U_{0}\right), \theta\right]\left(s-t_{0}\right) \\
& \leq D_{0}\left[\left(Q U_{0}\right), \theta\right]\left(t-t_{0}\right) \leq M_{1}\left(t-t_{0}\right) \leq M\left(t-t_{0}\right)=w_{0}(t),
\end{aligned}
$$

which implies that $D_{0}\left[U_{1}, U_{0}\right](t) \leq w_{0}(t)$. We assume, for some $k>1$,

$$
D_{0}\left[U_{k}, U_{k-1}\right](t) \leq w_{k-1}(t), \quad t \in J_{0} .
$$


Consider, for any $t \in J_{0}, t_{0} \leq s \leq t$,

$$
\begin{aligned}
D\left[U_{k+1}(s), U_{k}(s)\right] & \leq \int_{t_{0}}^{s} D\left[\left(Q U_{k}\right)(\xi),\left(Q U_{k-1}\right)(\xi)\right] d \xi \leq \int_{t_{0}}^{s} g\left(\xi, D_{0}\left[U_{k}, U_{k-1}\right](\xi)\right) d \xi \\
& \leq \int_{t_{0}}^{s} g\left(\xi, w_{k-1}(\xi)\right) d \xi \leq \int_{t_{0}}^{t} g\left(\xi, w_{k-1}(\xi)\right) d \xi=w_{k}(t),
\end{aligned}
$$

which further gives

$$
D_{0}\left[U_{k+1}, U_{k}\right](t) \leq w_{k}(t), \quad t \in J_{0} .
$$

Thus, we conclude that

$$
D_{0}\left[U_{n+1}, U_{n}\right](t) \leq w_{n}(t)
$$

for $t \in J_{0}$ and for all $n=0,1,2, \ldots$.

We claim that $\left\{U_{n}(t)\right\}$ is a Cauchy sequence. To show this, let $n \leq m$. Setting $v(t)=$ $D\left[U_{n}(t), U_{m}(t)\right]$ and using (3.19), we get

$$
\begin{aligned}
D^{+} v(t) \leq & D\left[D_{H} U_{n}(t), D_{H} U_{m}(t)\right](t)=D\left[\left(Q U_{n-1}\right)(t),\left(Q U_{m-1}\right)(t)\right] \\
\leq & D\left[\left(Q U_{n-1}\right)(t),\left(Q U_{n}\right)(t)\right]+D\left[\left(Q U_{n}\right)(t),\left(Q U_{m}\right)(t)\right] \\
& +D\left[\left(Q U_{m}\right)(t),\left(Q U_{m-1}\right)(t)\right] \leq g\left(t, D_{0}\left[U_{n-1}, U_{n}\right](t)\right)+g\left(t, D_{0}\left[U_{n}, U_{m}\right](t)\right) \\
& +g\left(t, D_{0}\left[U_{m-1}, U_{m}\right](t)\right) \leq g\left(t, w_{n-1}(t)\right)+g\left(t,|v|_{0}(t)\right)+g\left(t, w_{n-1}(t)\right) \\
= & g\left(t,|v|_{0}(t)\right)+2 g\left(t, w_{n-1}(t)\right) .
\end{aligned}
$$

The above inequalities yield, on using Theorem 3.2, the estimate

$$
v(t) \leq r_{n}(t), \quad t \in J_{0},
$$

where $r_{n}(t)$ is the maximal solution of

$$
r_{n}^{\prime}=g\left(t, r_{n}\right)+2 g\left(t, w_{n-1}(t)\right), \quad r_{n}\left(t_{0}\right)=0,
$$

for each $n$. Since as $n \rightarrow \infty, 2 g\left(t, w_{n-1}(t)\right) \rightarrow 0$ uniformly on $J_{0}$, it follows by [3, Lemma 1.3.1] that $r_{n}(t) \rightarrow 0$, as $n \rightarrow \infty$ uniformly on $J_{0}$. This implies from (3.30) that $U_{n}(t)$ converges uniformly to $U(t)$ on $J_{0}$ and clearly $U(t)$ is a solution of (3.2).

To prove uniqueness, let $V(t)$ be another solution of (3.2) on $J_{0}$. Set $m(t)=D[U(t)$, $V(t)]$. Then, $m\left(t_{0}\right)=0$ and

$$
D^{+} m(t) \leq g\left(t,|m|_{0}(t)\right), \quad t \in J_{0} .
$$

Since $m\left(t_{0}\right)=0$, it follows from Theorem 3.2 that

$$
m(t) \leq r\left(t, t_{0}, 0\right), \quad t \in J_{0},
$$


where $r\left(t, t_{0}, 0\right)$ is the maximal solution of (3.18). The assumption (b) now shows that $U(t)=V(t), t \in J_{0}$, proving uniqueness.

Assuming local existence, we next discuss a global existence result.

Theorem 3.6. Let $Q \in C[E, E]$ be a causal map such that

$$
D[(Q U)(t), \theta] \leq g\left(t, D_{0}[U, \theta](t)\right),
$$

where $g \in C\left[\mathbb{R}_{+}^{2}, \mathbb{R}_{+}\right], g(t, w)$ is nondecreasing in $w$ for each $t \in \mathbb{R}_{+}$and the maximal solution $r(t)=r\left(t, t_{0}, w_{0}\right)$ of (3.4) exists on $\left[t_{0}, \infty\right)$. Suppose further that $Q$ is smooth enough to guarantee the local existence of solutions of (3.2) for any $\left(t_{0}, U_{0}\right) \in \mathbb{R}_{+} \times K_{c}\left(\mathbb{R}^{n}\right)$. Then, the largest interval of existence of any solution $U\left(t, t_{0}, U_{0}\right)$ of $(3.2)$ is $\left[t_{0}, \infty\right)$, whenever $D\left[U_{0}, \theta\right] \leq w_{0}$.

Proof. Suppose that $U(t)=U\left(t, t_{0}, U_{0}\right)$ is any solution of (3.2) existing on $\left[t_{0}, \beta\right), t_{0}<\beta<\infty$ with $D\left[U_{0}, \theta\right] \leq w_{0}$, and the value of $\beta$ cannot be increased. Define $m(t)=D[U(t), \theta]$ and note that $m\left(t_{0}\right) \leq w_{0}$. Then, it follows that

$$
D^{+} m(t) \leq D\left[D_{H} U(t), \theta\right] \leq D[(Q U)(t), \theta] \leq g\left(t, D_{0}[U, \theta](t)\right) .
$$

Using Theorem 3.2, we obtain

$$
m(t) \leq r(t), \quad t_{0} \leq t<\beta .
$$

For any $t_{1}, t_{2}$ such that $t_{0}<t_{1}<t_{2}<\beta$, using (3.36) and the properties of Hausdorff metric $D$,

$$
\begin{aligned}
D\left[U\left(t_{1}\right), U\left(t_{2}\right)\right] & =D\left[\int_{t_{0}}^{t_{1}}(Q U)(s) d s, \int_{t_{0}}^{t_{2}}(Q U)(s) d s\right] \\
& \leq \int_{t_{1}}^{t_{2}} D[(Q U)(s), \theta] d s \leq \int_{t_{1}}^{t_{2}} g\left(s, D_{0}[U, \theta](s)\right) d s
\end{aligned}
$$

Employing the estimate (3.38) and the monotonicity of $g(t, w)$, we find

$$
D\left[U\left(t_{1}\right), U\left(t_{2}\right)\right] \leq \int_{t_{1}}^{t_{2}} g(s, r(s)) d s=r\left(t_{2}\right)-r\left(t_{1}\right) .
$$

Since $\lim _{t \rightarrow \beta^{-}} r\left(t, t_{0}, w_{0}\right)$ exists, taking the limit as $t_{1}, t_{2} \rightarrow \beta^{-}$, we get that $\left\{U\left(t_{n}\right)\right\}$ is a Cauchy sequence and therefore $\lim _{t \rightarrow \beta^{-}} U\left(t, t_{0}, U_{0}\right)=U_{\beta}$ exists. We then consider the IVP

$$
D_{H} U(t)=(Q U)(t), \quad U(\beta)=U_{\beta} .
$$

As we have assumed the local existence, we note that $U\left(t, t_{0}, U_{0}\right)$ can be continued beyond $\beta$, contradicting our assumption that $\beta$ cannot be increased. Thus, every solution $U\left(t, t_{0}, U_{0}\right)$ of $(3.2)$ such that $D\left[U_{0}, \theta\right] \leq w_{0}$ exists globally on $\left[t_{0}, \infty\right)$ and hence the proof follows.

Next, we will discuss the continuous dependence of solutions with respect to initial values. 
Lemma 3.7. Let $Q \in C[E, E]$ be a causal map and let

$$
G(t, k(t))=\sup \left[D[(Q U)(t), \theta]: D\left[U(t), U_{0}\right] \leq k(t)\right] .
$$

Assume that $r^{*}\left(t, t_{0}, 0\right)$ is the maximal solution of

$$
w^{\prime}=G(t, w), \quad w\left(t_{0}\right)=0, \quad \text { on } J .
$$

Let $U(t)=U\left(t, t_{0}, 0\right)$ be the solution of (3.2). Then,

$$
D\left[U(t), U_{0}\right] \leq r^{*}\left(t, t_{0}, 0\right), \quad t \in J .
$$

Proof. Set $m(t)=D\left[U(t), U_{0}\right], t \in J$. Then,

$$
\begin{aligned}
m(t+h)-m(t) & =D\left[U(t+h), U_{0}\right]-D\left[U(t), U_{0}\right] \\
& =D[U(t+h), U(t)+h(Q U)(t)]+D[U(t)+h(Q U)(t), U(t)] .
\end{aligned}
$$

Hence,

$$
\begin{gathered}
\frac{m(t+h)-m(t)}{h} \leq D\left[\frac{U(t+h)-U(t)}{h},(Q U)(t)\right]+D[(Q U)(t), \theta], \\
D^{+} m(t) \leq D[(Q U)(t), \theta] \leq \sup \left[D[(Q U)(t), \theta]: D\left[U(t), U_{0}\right] \leq m(t)\right] \leq G(t, m(t)) .
\end{gathered}
$$

This implies by [3, Theorem 1.4.1] that

$$
D\left[U(t), U_{0}\right] \leq r^{*}\left(t, t_{0}, 0\right), \quad t \in J .
$$

Theorem 3.8. Assume that

(a) assumptions (a), (b), and (c) of Theorem 3.5 hold;

(b) the solutions $w\left(t, t_{0}, w_{0}\right)$ of (3.4) through every point $\left(t_{0}, w_{0}\right)$ are continuous with respect to $\left(t_{0}, w_{0}\right)$.

Then, the solution $U(t)=U\left(t, t_{0}, U_{0}\right)$ of (3.2) is continuous with respect to $\left(t_{0}, U_{0}\right)$.

Proof. Let $U(t)=U\left(t, t_{0}, U_{0}\right), V(t)=V\left(t, t_{0}, V_{0}\right), U_{0}, V_{0} \in K_{c}\left(\mathbb{R}^{n}\right)$ be two solutions of (3.2). Then, defining $m(t)=D[U(t), V(t)]$, we get from Theorem 3.3 the estimate

$$
D[U(t), V(t)] \leq r\left(t, t_{0}, D\left[U_{0}, V_{0}\right]\right), \quad t \in J .
$$

Since $\lim _{U_{0} \rightarrow V_{0}} r\left(t, t_{0}, D\left[U_{0}, V_{0}\right]\right)=r\left(t, t_{0}, 0\right)$ uniformly on $J$ and by hypothesis $r\left(t, t_{0}, 0\right) \equiv$ 0 , consequently $\lim _{U_{0} \rightarrow V_{0}} U\left(t, t_{0}, U_{0}\right)=V\left(t, t_{0}, V_{0}\right)$ uniformly and hence $U\left(t, t_{0}, U_{0}\right)$ is continuous with respect to $U_{0}$.

To prove continuity with respect to $t_{0}$, we let $U(t)=U\left(t, t_{0}, U_{0}\right), V=V\left(t, \tau_{0}, U_{0}\right)$ be two solutions of (3.2) with $\tau_{0}>t_{0}$. Again, setting $m(t)=D[U(t), V(t)]$ and noting that $m\left(\tau_{0}\right)=D\left[U\left(\tau_{0}\right), U_{0}\right]$, using Lemma 3.7, we get

$$
m\left(\tau_{0}\right) \leq r^{*}\left(\tau_{0}, t_{0}, U_{0}\right)
$$




\section{Set differential equations with causal operators}

Hence, using Theorem 3.3, we obtain

$$
m(t)=\tilde{r}(t), \quad t \geq \tau_{0},
$$

where $\tilde{r}\left(t, \tau_{0}, r^{*}\left(\tau_{0}, t_{0}, 0\right)\right)$ is the maximal solution of (3.2) through $\left(\tau_{0}, r^{*}\left(\tau_{0}, t_{0}, 0\right)\right)$. Since $r^{*}\left(t, t_{0}, 0\right)=0$, we have

$$
\lim _{\tau_{0} \rightarrow t_{0}} \tilde{r}\left(t, \tau_{0}, r^{*}\left(\tau_{0}, t_{0}, 0\right)\right)=\tilde{r}\left(t, t_{0}, 0\right)
$$

uniformly on $J$. By hypothesis, $\tilde{r}\left(t, t_{0}, 0\right) \equiv 0$ which proves the continuity of $U\left(t, t_{0}, U_{0}\right)$ relative to $t_{0}$.

\section{References}

[1] C. Corduneanu, Functional Equations with Causal Operators, Stability and Control: Theory, Methods and Applications, vol. 16, Taylor \& Francis, London, 2002.

[2] V. Lakshmikantham, T. Gnana Bhaskar, and J. Vasundhara Devi, Theory of Set Differential Equations in a Metric Space, to appear.

[3] V. Lakshmikantham and S. Leela, Differential and Integral Inequalities: Theory and Applications. Vol. I. Ordinary Differential Equations, Academic Press, New York, 1969.

[4] A. Tolstonogov, Differential Inclusions in a Banach Space, Mathematics and Its Applications, vol. 524, Kluwer Academic Publishers, Dordrecht, 2000.

Z. Drici: Department of Mathematics and Computer Science, Illinois Wesleyan University, Bloomington, IL 61702-2900, USA

E-mail address: zdrici@iwu.edu

F. A. Mcrae: Department of Mathematics, Catholic University of America, Washington, DC 20064, USA

E-mail address: mcrae@cua.edu

J. Vasundhara Devi: Department of Mathematics, GVP College for PG Courses, Andhra University, Visakhapatnam, India

Current address: Department of Mathematical Sciences, Florida Institute of Technology, Melbourne, FL 32901, USA

E-mail address: jdevi@fit.edu 


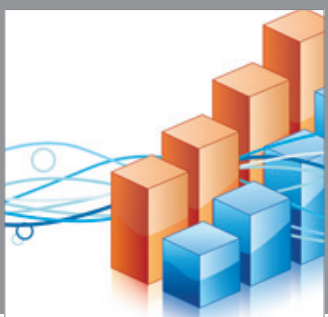

Advances in

Operations Research

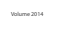

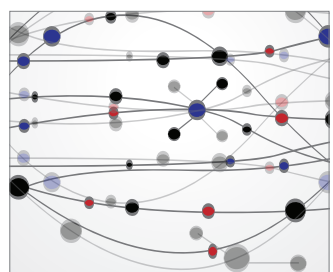

\section{The Scientific} World Journal
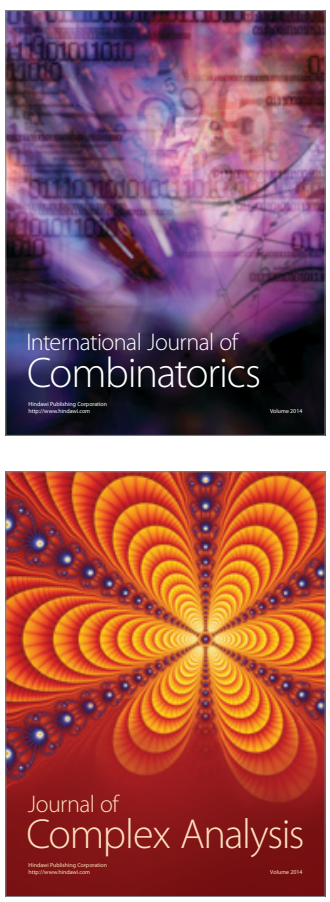

International Journal of

Mathematics and

Mathematical

Sciences
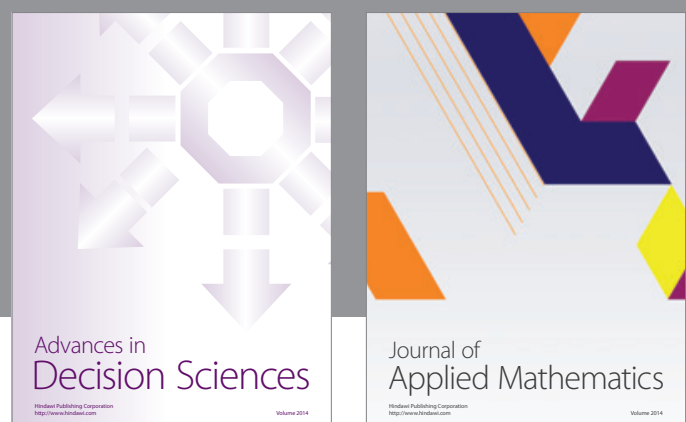

Journal of

Applied Mathematics
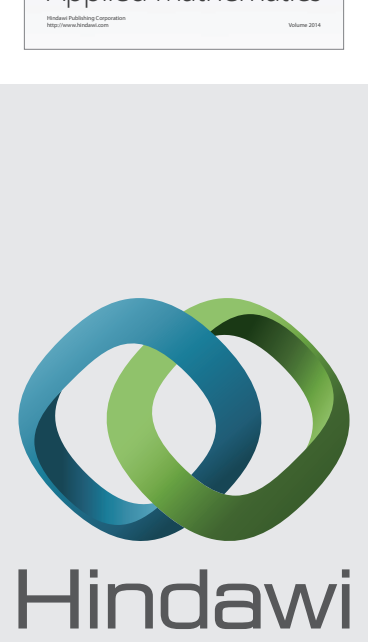

Submit your manuscripts at http://www.hindawi.com
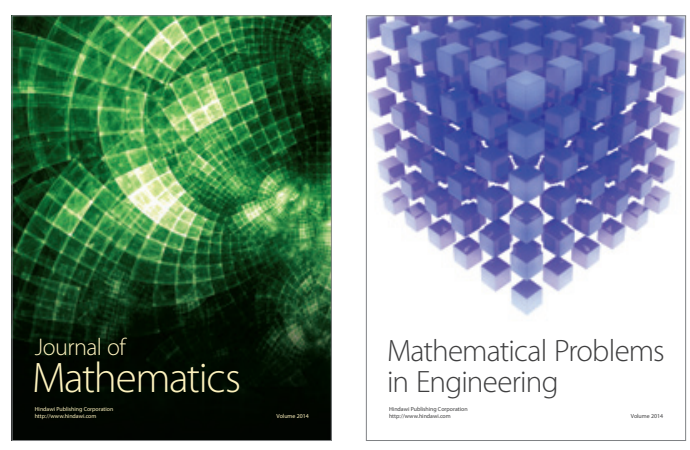

Mathematical Problems in Engineering
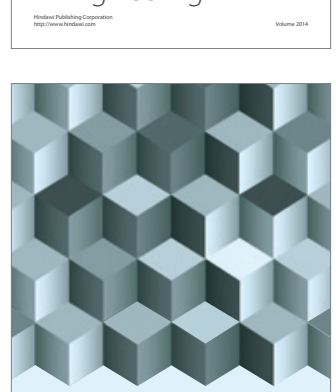

Journal of

Function Spaces
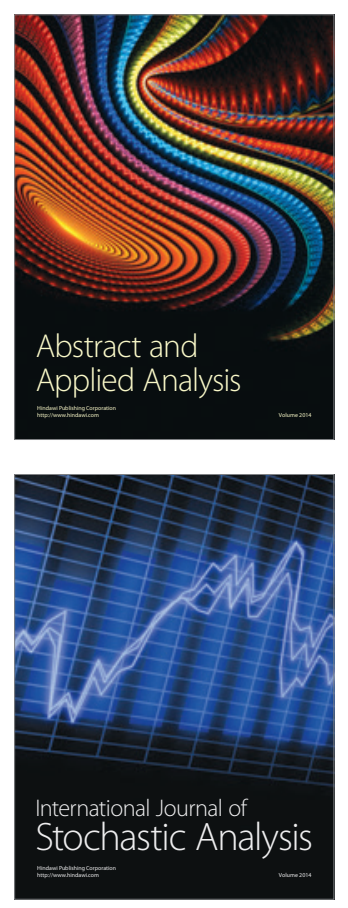

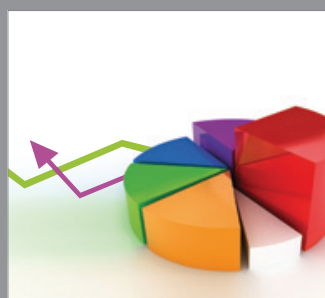

ournal of

Probability and Statistics

Promensencen
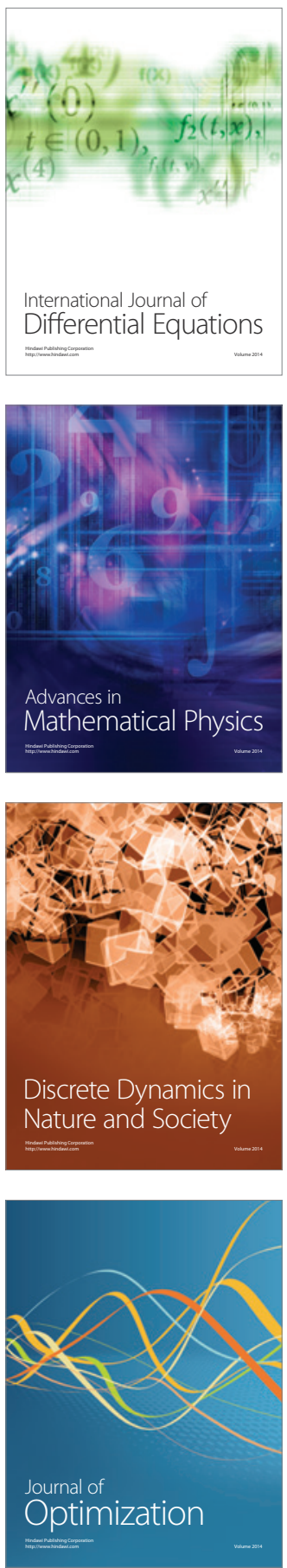